Atherosclerosis. 2018 November ; 278: 39-48. doi:10.1016/j.atherosclerosis.2018.09.018.

\title{
Krüppel-like factor 14, a coronary artery disease associated transcription factor, inhibits endothelial inflammation via NF- $x B$ signaling pathway
}

\author{
Wenting Hu${ }^{a, b}$, Haocheng Lu ${ }^{a}$, Jifeng Zhang ${ }^{a}$, Yanbo Fan ${ }^{a}$, Ziyi Chang ${ }^{a}$, Wenying Liang ${ }^{a}$, \\ Huilun Wang ${ }^{\mathrm{a}}$, Tianqing Zhu ${ }^{\mathrm{a}}$, Minerva T. Garcia-Barrio ${ }^{\mathrm{a}}$, Daoquan Peng ${ }^{\mathrm{b}}$, Y. Eugene Chen ${ }^{\mathrm{a}}$, \\ and Yanhong Guo ${ }^{a}$ \\ ${ }^{a}$ Cardiovascular Center, Department of Internal Medicine, University of Michigan Medical Center, \\ Ann Arbor, MI 48109, USA \\ bDepartment of Cardiovascular Medicine, the Second Xiangya Hospital, Central South University, \\ Changsha, 410011, China
}

\begin{abstract}
Background and aims: Human genetic studies indicated that variations near the transcription factor Krüppel-like factor 14 (KLF14) gene locus were highly associated with coronary artery disease. Activation of endothelial cells (ECs) by pro-inflammatory molecules and pathways is a primary step in atherosclerosis development. We aimed to investigate the effects and mechanism of KLF14 on inflammatory responses in ECs.
\end{abstract}

Methods: Adenovirus-mediated overexpression of human KLF14 and EC specific KIf14 knockout mice were applied to study the role of KLF14 in the EC inflammation. Intravital microscopy was used to examine leukocyte-endothelial cell interactions in vivo.

\begin{abstract}
Results: The expression of Klf14 was markedly decreased in mouse aortic ECs in both acute and chronic inflammatory conditions. Overexpression of KLF14 inhibited inflammatory activation of human ECs stimulated by interleukin $1 \beta$ and tumor necrosis factor $a$. Primary pulmonary ECs from Klf14 knockout mice showed increased expression of adhesion molecules under IL-1 $\beta$ stimuli. Mechanistically, KLF14 inhibited NF- $\mathrm{KB}$ signaling pathway by transcriptionally suppressing the expression of p65, resulting in significantly decreased leukocyte adhesion to activated ECs. Using intravital microscopy, an increased leukocyte-endothelial cell interaction was observed in endothelial specific Klfl4 knockout mice compared to wild type control mice.
\end{abstract}

\footnotetext{
Corresponding authors: Yanhong Guo, MD, PhD, University of Michigan Medical Center, NCRC Bld 26, Rm 355S, 2800 Plymouth Rd, Ann Arbor, MI 48109, Phone: 734-647-5750, Fax: 734-763-7097, yanhong @ umich.edu, or, Y. Eugene Chen, MD, PhD,

University of Michigan Medical Center, NCRC Bld 26, Rm 361S, 2800 Plymouth Rd, Ann Arbor, MI 48109, Phone: 734-647-5742, Fax: 734-763-7097, echenum@umich.edu.

Author distributions:

Wenting Hu designed the experiment, acquired the data, interpreted the result and drafted the manuscript. Haocheng Lu contributed to the molecular biology work, intravital microscopy and polishing the manuscript. Jifeng Zhang provided essential support to the animal study and molecular cloning work. Yanbo Fan, Ziyi Chang, Wenying Liang, Huilun Wang, Tianqing Zhu provided extensive support to the experiment. Minerva T. Garcia-Barrio helped to discuss the result and contribute to the manuscript. Daoquan Peng, Yanhong Guo and Y. Eugene Chen conceived the idea and coordinated the experiments. All authors read and approved the final manuscript.

Conflict of Interest

The authors declare no actual or potential conflicts of interest.
} 
Additionally, perhexiline, a KLF14 activator, induces KLF14 expression in ECs and reduced leukocyte-endothelial cell interactions in vitro and in vivo.

Conclusions: The data revealed that KLF14 inhibited the inflammatory response in ECs and the protective effects were mediated by transcriptional inhibition of NF- $\mathrm{KB}$ signaling pathway. Endothelial KLF14 could be a potential therapeutic target for cardiovascular diseases.

\section{Keywords}

Krüppel-like factor 14; endothelial cell; inflammation; transcription

\section{Introduction}

Atherosclerosis, characterized by build-up of atherosclerotic plaque in the blood vessels, is a common pathological process shared by most of cardiovascular diseases, including coronary artery disease (CAD), ischemic stroke and peripheral arterial disease. Despite the enormous effort in basic and clinical studies, cardiovascular diseases are still the leading global cause of mobility and mortality. Human genetic studies have identified more than 100 loci that are associated to $\mathrm{CAD}^{1}$. Confirmed causal relationships lead to new mechanisms and therapeutic targets of atherosclerosis such as the successful application of mono-antibody against proprotein convertase subtilisin/kexin type 9 (PCSK9) ${ }^{2}$. However, the precise mechanism underlying most of the $\mathrm{CAD}$ associated loci remains elusive.

Atherosclerosis is a chronic inflammatory disease and endothelial cell (EC) inflammatory activation/dysfunction takes place in the early phase of atherogenesis ${ }^{12}$. Endothelial cells, the inner layer of the vascular wall, serve as important regulators of vascular inflammation, blood aggregation, vascular tone and capillary permeability. Quiescent endothelial cells express none or low level of adhesion molecules and are resistant to leukocyte recruitment. However, a variety of pro-atherogenic stimuli, such as tumor necrosis factor a (TNFa), interleukin $1 \beta$ (IL-1 $\beta$ ) or oxidized lipoproteins, can activate the endothelial inflammatory cascade, mainly through nuclear factor kappa B (NF- $\mathrm{kB}$ ) pathway. These factors initiate the transcription of adhesion molecules (vascular cell adhesion protein 1 (VCAM-1), endothelial selectin (E-selectin), intercellular adhesion molecule 1(ICAM-1)) as well as chemokines (monocyte chemotactic protein 1 (MCP1) and IL-1 $\beta$ ) in $\mathrm{ECs}^{13}$, followed by inflammatory cells rolling, adhering and infiltrating across the endothelial layer into the vascular wall. Infiltrated monocytes differentiate to macrophages in the vascular wall and uptake oxidized low density lipoprotein particles turn into foam cells ${ }^{14}$. Accumulating cholesterol in atherosclerotic plaques can further promote local inflammation, forming a vicious cycle ${ }^{15}$. Recently, a Novartis Phase III study showed that IL- $1 \beta$ antibody, Canakinumab, reduces cardiovascular risk in individuals who survived a heart attack (the CANTOS Trial), reinforcing that anti-inflammatory therapy could successfully prevent $\mathrm{CAD}^{16}$. Consider that inflammatory response might be important under physiological conditions, such as attracting monocytes to remove extra cholesterol in the sub-endothelial layer, targeting the pathogenic downstream effects of vascular endothelial cells, instead of blocking the production of inflammatory molecules, may become better anti-inflammatory therapies against hyperlipidemia-induced endothelial cell activation. 
Genome-wide association studies involving $>100,000$ individuals found that a variant near the $K L F 14$ region (rs4731702) is associated with high-density lipoprotein cholesterol and $\mathrm{CAD}$, a finding confirmed by other independent studies ${ }^{3-5}$. Krüppel-like factors (KLFs) are zinc finger DNA-binding proteins. There are 17 members already identified in the mammalian genome. KLFs have been shown to regulate cell proliferation, differentiation and apoptosis in a variety of diseases, including cardiovascular diseases, metabolic diseases and cancers ${ }^{6}$. Among them, KLF14 was first cloned in 2000 and is ubiquitously expressed in mouse organs ${ }^{7}$. KLF14 is implicated in regulating T-regulatory cell differentiation ${ }^{8}$, tumorigenesis ${ }^{9}$, and metabolic phenotypes ${ }^{10}$. Using a liver-specific Klf 14 deletion mouse model, our lab demonstrated that activation of KLF14 reduces atherosclerosis in apolipoprotein $\mathrm{E}$ deficient mice by modulating apolipoprotein A1 production ${ }^{11}$.

To further explore the underlying mechanism of KLF14 in vascular wall homeostasis, both gain-of-function and loss-of-function studies were used to delineate its function in endothelial cells. Here, we found that KLF14 was suppressed by pro-inflammatory factors while KLF14 overexpression inhibited EC inflammation by reducing p65 transcription. Using EC-specific Klf14 knockout (KO) mouse, we demonstrated that Klf14 deficiency promoted leukocyte-endothelial adhesion in vivo, supporting an anti-inflammatory role for KLF14 in ECs.

\section{Materials and Methods}

\subsection{Animals and Cells}

Klf14flox/flox mice ${ }^{11}$ were mated with Cdh5-Cre transgenic mice (stock number: 006137, Jackson Laboratory) to generate endothelial specific KIf14 KO mice. Six to eight weeks old male Cdh5-Cre/KIf14 flox/flox mice were used for in vivo leukocyte adhesion assay. KIf14 global KO mice were generated by crossing Klf14 flox/flox mice with Ayu1-Cre mice that express Cre ubiquitously ${ }^{17}$. Blood pressure were measured via tail-cuff method using BP-2000 blood pressure analysis system (Visitech technology). The complete blood count was analyzed by In-Vivo animal core of University of Michigan with Hemavet 950 machine (Drew Scientific). All animal procedures followed guidelines of the University of Michigan Animal Care and Use Committee.

Human coronary artery endothelial cells (HCAECs) (CC2585, from a 37-year old male donor) and Human umbilical vein endothelial cells (HUVECs) (C2519, pooled donors) were purchased from Lonza. Mouse pulmonary endothelial cells were isolated by two rounds of cell sorting using CD31 and ICAM-2 antibody coated magnetic beads.

\subsection{In vitro leukocyte endothelial adhesion assay}

HUVECs were infected with 10 MOI AdLacZ or AdKLF14 for 48 hours before stimulated with proinflammatory cytokines for 6 hours. Simultaneously, human monocytes, THP-1 cells, were incubated with fluorescence-labelled LeukoTracker (Cell Biolabs, CytoSelect ${ }^{\mathrm{TM}}$ Leukocyte- Endothelium Adhesion Assay kit, CBA-210) for 1 hour at $37^{\circ} \mathrm{C}$ and washed according to the manufacturer's protocol. Fluorescence-labelled THP-1 cells were coincubated with control or activated HUVECs for $30 \mathrm{~min}$. Then, the unbound THP-1 cells 
were removed by washing. The number of adhered cells was counted under a fluorescence microscope.

\subsection{Intravital microscopy}

Intravital microscopy analysis was performed as previously described ${ }^{18}$. There were two experimental approaches in this study. In the first experiment, 6-8 weeks old male ECspecific KIf14 KO mice and littermate wild type mice were injected intraperitoneally with 5 $\mathrm{mg} / \mathrm{Kg}$ LPS. In the second experiment, 6-8 weeks old male wild type mice on C57BL/6 background and EC-specific Klf14 KO mice were injected intraperitoneally with DMSO or perhexiline maleate salt at $10 \mathrm{mg} / \mathrm{Kg} / \mathrm{d}$ every other day at least three times before $10 \mathrm{mg} / \mathrm{Kg}$ LPS injection. Four hours after LPS administration, mice were anesthetized and injected with rhodamine $6 \mathrm{G}$ chloride to stain leukocytes. The cremaster muscle was dissected and vessel wall-leukocyte interaction in post-capillary venules were observed using an intravital microscope. Leukocytes that remain stationary for at least 30 s were counted to be adherent to vascular endothelial cells.

Materials and Methods in details are available in the supplementary files.

\section{Results}

\subsection{Endothelial KIf14 is down-regulated in acute and chronic inflammatory conditions}

Endothelial inflammation is a hallmark of endothelial dysfunction and is the driving force for the development of the atherosclerotic plaque. Since $K L F 14$ has been revealed as a CAD associated gene, we first aimed to assess if KLF14 expression was altered in inflammatory conditions. To achieve this, we induced an acute inflammatory model using adult C57BL/6J mice by lipopolysaccharides (LPS) injection, as well as a chronic inflammatory model by challenge mice with western diet for 3 months. We observed a dramatically decreased intensity of KLF14 in aortic endothelial cells by immunofluorescence staining (Fig. 1A and B) and reduced Klf14 mRNA levels in the aorta by qRT-PCR (Fig. 1C) in LPS-treated mice. Due to a relatively low level of expression in smooth muscle cells as compared with the endothelial cells in mouse aorta, we considered that the expression of Klf14 in the aorta tissue mainly represented the levels in endothelial cells after we carefully removed adventitia. C57BL/6J mice are susceptible to western diet-induced obesity and metabolic syndrome ${ }^{19}$. We found similar expression changes both in protein and mRNA levels (Fig. 1D-F). Lung is a vascular-rich organ, so we also determined Klf14 expression in mouse lung tissue. Results showed that both of Klf14 mRNA and protein abundance were reduced in mouse lung tissues in both LPS treated and western diet challenged mice (Supplement Fig. $1 \mathrm{~A}-\mathrm{D})$. Consistent with the in vivo mouse results, we observed that IL- $1 \beta$ treatment could reduce $K L F 14$ mRNA levels in cultured human umbilical vein endothelial cells (HUVECs) (Fig. $1 \mathrm{G}$ and $\mathrm{H}$ ). These data indicate that KLF14, a CAD associated gene, was decreased in ECs under inflammatory stimuli, suggesting that KLF14 potentially contributes to EC inflammatory response. 


\subsection{KLF14 overexpression inhibits EC inflammation}

EC inflammation is characterized by the induction of a variety of inflammatory cytokines and adhesion molecules, e.g. VCAM-1 and E-selectin. Two kinds of primary cultured human endothelial cells were used to determine the role of KLF14 on EC inflammation. Both of human coronary artery endothelial cells (HCAECs) and HUVECs are well-established in vitro model to study endothelial cell biology ${ }^{20}$, which were stimulated with the proinflammatory cytokines IL-1 $\beta$ and TNFa, since both of these cytokines are involved in the development of atherosclerosis ${ }^{21,22}$. Adenovirus-mediated KLF14 overexpression potently inhibited VCAM-1 and E-selectin mRNA (Fig. 2A and D) and protein (Fig. 2B, C, E and F) abundance in both HCAECs and HUVECs stimulated by IL- $1 \beta$ or TNFa. Moreover, increased KLF14 level gradually blocked VCAM- 1 and E-selectin induction by IL-1 $\beta$ in a dose dependent manner (Supplement Fig. 2) in HUVECs, indicating that KLF14 overexpression is sufficient to inhibit the expression of adhesion molecules in human ECs.

\subsection{KIf14 deficient endothelial cells express increased inflammatory markers}

To determine whether KLF14 is essential for EC inflammatory regulation, we generated global Klf14 deficient mice (with Ayu1-cre) (Supplement Fig. 3A) and isolated pulmonary endothelial cells from both KIf14 flox/KIf14flox (wild type, WT) and Ayu1-cre+ Klf14flox/ KIf14 $4^{\text {flox }}$ mice (KIf14 KO mice) by sorting the cells with CD31 and ICAM-2 antibodycoated beads $^{23}$ (Supplement Fig. 3B). ECs purity was over $80 \%$ of total cells detected by Dil-labeled acetylated low density lipoprotein uptake assay and Von Willebrand factor staining ${ }^{24}$ (Supplement Fig. 3C and D). Klf14 deficiency was confirmed by qRT-PCR (Supplement Fig. 3E). Klf14-deficient endothelial cells exhibited increased VCAM-1 and Eselectin expression upon IL-1 $\beta$ stimulation (Fig. $2 \mathrm{G}, \mathrm{H}$ and I). We also assessed the expression of genes involved in thrombosis, metabolism and ER stress (Supplement Fig. 3F) between WT and Klf14-deficient endothelial cells. Among them, we also found that Klf14 deficiency increases tissue factor expression. Importantly, deficiency of Klf14 did not affect the expression of Klf2, Klf4 or Klf11, so the anti-inflammatory effect of KLF14 was not due to compensation of other KLF members (Supplement Fig. 4). Therefore, KLF14 is a negative regulator of EC inflammation.

\subsection{KLF14 ameliorates endothelial inflammation by inhibiting NF- $\mathrm{KB}$ signaling pathway}

NF- $\kappa B$ signaling pathway is the most important pathway regulating EC inflammation. Both VCAM-1 and E-selectin are direct targets of NF- $\mathrm{kB}$ signaling pathway ${ }^{25-27}$. In bovine aortic endothelial cells (BAECs), which are commonly used for plasmid transduction in endothelial biology studies ${ }^{28}$, we found that KLF14 overexpression reduced TNFa- induced luciferase activity using a reporter containing NF- $\kappa B$ response elements, indicating that KLF14 might negatively regulate NF- $\kappa$ B pathway (Supplement Fig. 5A). Indeed, administration of NF- $\kappa$ B pathway inhibitors (BAY 11-7082, which inhibits phosphorylation of NF- $\kappa \mathrm{B}$ inhibitor a (I $\kappa \mathrm{Ba})$ and ACHP, which blocks I $\kappa \mathrm{B}$ kinase activity) completely abolished the anti-inflammatory function of KLF14 in HUVECs (Supplement Fig. 5B-E). As a result, we considered that KLF14 inhibited EC inflammation in an NF- $\mathrm{KB}$-dependent fashion. 


\subsection{KLF14 inhibits transcription factor p65 (p65) transcription}

The activation of NF- $\kappa \mathrm{B}$ signaling pathway requires the degradation of NF- $\kappa \mathrm{B}$ inhibitor a $(\mathrm{I} \kappa \mathrm{Ba}$ ) to ensure the nuclear translocation of transcription factor p65 (also known as RELA). To delineate how KLF14 regulates NF- $\kappa B$ signaling pathway, we assessed NF- $\kappa B$ inhibitor alpha degradation and p65 nuclear translocation. Intriguingly, KLF14 overexpression resulted in reduced p65 both in cytoplasm and nucleus upon IL-1 $\beta$ stimulation (Fig. 3A and Supplement Fig. 6A) but did not inhibit NF-кB inhibitor alpha degradation (actually, it is slightly increased) (Supplement Fig. 6B). This novel finding is consistent with KLF14 overexpression in HUVECs decreasing p65 mRNA and protein (Fig. 3B, C and D). Pulmonary EC from Klf14 KO mice showing higher levels of p65 mRNA and protein upon IL-1 $\beta$ stimulation (Fig. 3E, F and G). Additionally, KLF14 downregulated luciferase expression driven by the SELE or VCAM1 promoters and this effect was abolished by p65 overexpression, showing that p65 mediated KLF14 function in ECs (Fig. $3 \mathrm{H})$. Considering that KLF14 is a transcription factor, we subsequently determined whether KLF14 could directly modulate p65 transcription. There are 2 CACCC core motifs located in the p65 promoter near the transcription start site (-359/-355 and $-179 /-175)$. Through chromatin immunoprecipitation assay we determined that KLF14 directly binds to these two regions (Fig. 3I). Using p65 promoter driving luciferase, we demonstrated that KLF14 inhibited p65 transcription and mutation of either CACCC motif diminished this effect (Fig. $3 J)$. In addition, although KLF2, KLF4 and KLF11 all exert anti-inflammatory effects in ECs, they do not directly inhibit transcription of endogenous p65 (Supplement Fig. 7), underscoring the distinct role of KLF14 in EC inflammation. As a result, we found a novel pathway by which KLF14 inhibits EC inflammation via repression of p65 transcription.

\subsection{KLF14 reduces leukocyte-endothelial adhesion}

Activated endothelial cells express high level of adhesion molecules on their surface, resulting in increased leukocyte adhesion to the vascular wall. Next we performed leukocyte adhesion assays both in vitro and in vivo to investigate whether KLF14 contribution to this process ${ }^{18}$. In Fig. 4A and B, KLF14 overexpression in HUVECs significantly reduced adhesion of THP-1 (human monocytic cell line labelled with green fluorescence) to EC after IL-1 $\beta$ or TNFa stimulation. For the in vivo assay, we generated endothelial-specific Klf14 KO (Klf14 EC KO) mice by crossbreeding Klf14 flox $/ K I f 14^{\text {flox }}$ mice with vascular endothelial cadherin 5 ( $C d h 5$ ) promoter driving-cre-recombinase (cdh5-cre) transgenic mice (Supplement Fig. 8A). Klf14 KO did not influence blood pressure or circulating leukocytes (Supplement Fig. 8B and C). Intravital microscopy is a powerful and widely used tool to study the blood cells and endothelium interaction in the mouse cremaster muscle in vivo. We observed a significantly increased leukocyte adhesion to endothelial cells in Klf14 EC KO mice compared to Cdh5-cre negative control mice (Klf14flox $/$ Klf14flox mice) (Fig. 4C and 4D). These data indicate that KLF14 inhibits leukocyte adhesion and is a novel factor regulating vascular wall homeostasis. 


\subsection{Perhexiline inhibits endothelial inflammation in an endothelial KLF14 dependent manner}

Previously, our lab identified perhexiline as a KLF14 activator after high throughput screening ${ }^{11}$. We also confirmed that perhexiline upregulated KLF14 mRNA in HUVECs (Fig. 5A). Pretreating HUVECs with perhexiline significantly inhibited IL-1 $\beta$ induced upregulation of VCAM-1 and E-selectin expression in both of mRNA and protein levels (Fig. 5B, C and D). To further address the role of perhexiline on $\mathrm{EC}$ inflammation in vivo, we pretreated mice with perhexiline via intraperitoneal injection to increase KLF14 expression and then assessed leukocyte-endothelial adhesion using intravital microscopy. Consistent with the in vitro data, perhexiline injection reduced leukocyte adhesion in wild type mice. To further detect whether pharmacological inhibition of inflammation effect of perhexiline was dependent on KLF14 in endothelial cells, we performed leukocyteendothelial adhesion using Klf14 EC KO mice. Notably, the anti-inflammatory effect of perhexiline was abolished in Klf14 EC KO mice compared to the control mice, demonstrating that perhexiline inhibits inflammatory response and leukocyte adhesion in ECs at least partially through inducing KLF14 (Fig. 5E and F). These results further demonstrate the key role of the KLF14 in endothelial inflammatory response and related cardiovascular diseases. The effect and mechanism of KLF14 on EC inflammatory response was showed in Fig. 5G.

\section{Discussion}

KLF14, a transcription factor, is associated with CAD, type 2 diabetes and cancer identified by human genetic studies. While the role of KLF14 in the liver has been elucidated in recent studies, the function of KLF14 in vascular cells has yet to be determined. Considering the importance of ECs in vascular homeostasis, molecule mechanisms of EC inflammation require more detailed studies. In this study, we found that endothelial KLF14 is an important negative regulator of inflammation. Klf14 expression is downregulated in both acute and chronic inflammatory conditions. The downregulated expression of KLF14 in endothelial cells in both acute and chronic inflammatory conditions suggests an insufficient effect of KLF14 in inflammatory response. Iwaya et al also reported that methylation level of KLF14 is increased in the adipose tissue after HFD challenge and this epigenetic change is significantly associated with inflammatory levels ${ }^{29}$. Restoration of KLF14 in human endothelial cells by adenovirus-mediated overexpression inhibits the upregulation of adhesion molecules induced by pro-inflammatory factors. This phenomenon further drives us to study if endogenous KLF14 is required for EC function. Using EC-specific Klf14 KO mice, we demonstrated that EC Klf14 deficiency promotes leukocyte adhesion in vivo. Here, we also showed that perhexiline activates Klf14 in ECs and reduced EC inflammatory response in vitro and in vivo.

Activation of NF- $\mathrm{BB} /$ Rel transcription factors plays a central role in endothelial inflammation. Stimulation of innate immune receptors or cytokine receptors results in activation of I $\kappa \mathrm{B}$ kinase (IKK). IKK phosphorylates I $\kappa \mathrm{B} a$ driving its degradation. Released

$\mathrm{NF}-\mathrm{\kappa B}$ can translocate to nucleus and initiate the transcription of targeted genes ${ }^{30}$. There are 5 members in NF- $\mathrm{BB}$ family — RelA (p65), RelB, c-Rel, p50 (p105 precursor) and p52 
(p100 precursor). They can form homo- or heterodimers to become a transcriptional activator and p50-p65 is most common among the various dimeric combinations ${ }^{31}$. Although the regulation of $\mathrm{p} 65$ nuclear translocation has been extensively studied, the transcriptional regulation of $\mathrm{p} 65$ is poorly understood ${ }^{32}$. p65 transcription is known to be activated by $\mathrm{Sp}^{33}$ and repressed by zinc finger and BTB domain containing 2 (ZBTB2) ${ }^{34}$. Our study found that KLF14 inhibited $N F-\kappa B$ reporter luciferase and this effect was abolished by I $\kappa \mathrm{Ba}$ phosphorylation inhibitors, indicating that the inhibition took place upstreaming of p65 nuclear translocation. However, we did not find evidence that KLF14 reduced I $\mathrm{KBa}$ degradation and this phenomenon lead us to uncover that KLF14 directly inhibited p65 transcription. p65 promoter contains high GC regions near the transcription start site, which are preferred binding sites for KLF proteins. Using ChIP assay, we demonstrated that KLF14 binds to the proximal region of the $\mathrm{p} 65$ promoter and repress its transcription, resulting in decreased p65 protein abundance in both cytoplasm and nucleus and inhibited inflammatory response in ECs.

Seventeen members of KLF family proteins exert a variety of functions in different cell types. Studies from our group and others indicate that KLF2, 4 and 11 are implicated in EC inflammation. Although NF- $\mathrm{KB}$ signaling pathway is the common target in KLF2, 4, and 11 studies, they regulate NF- $\kappa \mathrm{B}$ activities at different levels. KLF2 and KLF4 inhibit EC proinflammatory activation by recruiting transcriptional coactivator cyclic AMP response element-binding protein (CBP/p300) $)^{35,36}$ and prevent its binding to $\mathrm{p} 65$. KLF11 suppresses EC activation via physical interaction with $\mathrm{p} 65^{37}$. Hao et al reported that thrombin upregulates KLF14 expression and KLF14 modulates cellular oxidation state through Pololike kinase 1 in $\mathrm{ECs}^{38}$. Here, we identified that KLF14 could inhibit EC inflammation through a distinct mechanism involving inhibition of p65 expression. In addition, we also demonstrated that KLF2, KLF4 and KLF11 could not directly regulate p65 mRNA level, indicating a unique mechanism of KLF14 in regulation of EC inflammation.

In the in vitro studies, we used adenovirus mediated overexpression to study KLF14 function in human endothelial cells. There are certain limitations about the forced high expression of protein in the cell, for example, it would be non-physiological or potential toxic to cells ${ }^{39}$. To overcome this, we isolated Klf14-deficient endothelial cell from mice to confirm the role of Klf14. In addition, because of the anti-inflammatory role of KLF14 in endothelial cells, it is interesting to further investigate if Klf14 KO would affect atherosclerosis development in ApoE or Ldlr KO background.

Although lowering low-density lipoprotein cholesterol (LDL-C) level has significantly reduced CAD events ${ }^{40,41}$, novel therapies are still urgent in the field even with optimal statins treatment ${ }^{42}$. The benefits of the anti-inflammatory treatment in CAD patients suggest that this is a valid mechanism ${ }^{16}$. Increasing understanding of the effect and underlying mechanism of KLF14 in EC inflammation will uncover promising therapeutic target. Perhexiline is an antianginal drug, acting by inhibiting mitochondrial carnitine palmitoyltransferase- 1 to reduce fatty acid metabolism and increase myocardial efficiency per unit oxygen consumption. ${ }^{32}$ In a previous publication, our lab identified perhexiline as a KLF14 activator independent of its function in mitochondria ${ }^{11}$. Here we show that administration of perhexiline reduced leukocyte-endothelial adhesion in vivo and this 
protective effect disappeared in EC-specific Klf14 KO mice, demonstrating a KLF14dependent effect on EC inflammatory activation. There may be difference between human and mouse pharmacodynamics and pharmacokinetics of perhexiline and whether perhexiline would have similar anti-inflammatory effect in human requires further studies using humanderived cells or clinical data.

Based on these results, we provide a reasonable evidence that KLF14 contributes to vascular pathology in the aspect of inhibition of ECs inflammation. KLF14 inhibits EC inflammation by direct repression of p65 transcription. In the context of the human genetic studies, this finding further indicates that KLF14 could be a therapeutic target to treat CAD.

\title{
Supplementary Material
}

Refer to Web version on PubMed Central for supplementary material.

\section{Acknowledgments}

\author{
Financial support: \\ This work was supported in whole or in part by NIH grants HL068878, HL134569, and HL137214 (to Y.E. Chen) \\ and by American Heart Association 14SDG19880014 (to Y. Fan), 15SDG24470155 (to Y. Guo), 17PRE33400179 \\ (to H. Lu), and by Rackham Graduate School, University of Michigan U016449 (to H. Wang) and by National \\ Natural Science Foundation of China No. 81370393 and No. 81670426 (to D. Peng).
}

\section{Abbreviations:}

KLF14

EC

CAD

IL-1 $\beta$

TNF a

HCAECS

HUVECS

VCAM-1

E-selectin

ICAM-1

MCP-1

LPS

CD

WD
Krüppel-like factor 14

endothelial cell

coronary artery disease

interleukin $1 \beta$

tumor necrosis factor a

Human coronary artery endothelial cells

Human umbilical vein endothelial cells

vascular cell adhesion protein 1

endothelial selectin

intercellular adhesion molecule 1

monocyte chemotactic protein 1

lipopolysaccharides

chow diet

western diet 


$\begin{array}{ll}\text { NF- } x \text { B } & \begin{array}{l}\text { nuclear factor kappa-light-chain-enhancer of activated B } \\ \text { cells }\end{array} \\ \text { p65 } & \text { nuclear factor NF-kappa-B p65 subunit } \\ \text { ChIP } & \text { chromatin immunoprecipitation } \\ \text { KO } & \text { knockout }\end{array}$

\section{References:}

[1]. Khera AV and Kathiresan S, Genetics of coronary artery disease: discovery, biology and clinical translation, Nat Rev Genet, 2017;18:331-344. [PubMed: 28286336]

[2]. Shapiro MD, Tavori H and Fazio S, PCSK9: From Basic Science Discoveries to Clinical Trials, Circ Res, 2018;122:1420-1438. [PubMed: 29748367]

[3]. Teslovich TM, Musunuru K, Smith AV, et al., Biological, clinical, and population relevance of 95 loci for blood lipids, Nature, 2010;466:707. [PubMed: 20686565]

[4]. Huang P, Yin R-X, Huang K-K, et al., Association of the KLF14 rs4731702 SNP and serum lipid levels in the Guangxi Mulao and Han populations, BioMed research international, 2013;2013.

[5]. Guo Y, Garcia-Barrio MT, Wang L, et al., Experimental Biology for the Identification of Causal Pathways in Atherosclerosis, Cardiovascular Drugs and Therapy, 2016;30:1-11. [PubMed: 26847647]

[6]. Fan Y, Lu H, Liang W, et al., Krüppel-like factors and vascular wall homeostasis, Journal of Molecular Cell Biology, 2017:1-12. [PubMed: 28130307]

[7]. Scohy S, Gabant P, Van Reeth T, et al., Identification of KLF13 and KLF14 (SP6), novel members of the SP/XKLF transcription factor family, Genomics, 2000;70:93-101. [PubMed: 11087666]

[8]. Sarmento OF, Svingen PA, Xiong Y, et al., A novel role for Kruppel-like Factor 14 (KLF14) in Tregulatory cell differentiation, CMGH Cellular and Molecular Gastroenterology and Hepatology, 2015;1:188-202. e184. [PubMed: 25750932]

[9]. Fan G, Sun L, Shan P, et al., Loss of KLF14 triggers centrosome amplification and tumorigenesis, Nature communications, 2015;6:8450.

[10]. Consortium M, Identification of an imprinted master trans regulator at the KLF14 locus related to multiple metabolic phenotypes, Nature genetics, 2011;43:561-564. [PubMed: 21572415]

[11]. Guo Y, Fan Y, Zhang J, et al., Perhexiline activates KLF14 and reduces atherosclerosis by modulating ApoA-I production, J Clin Invest, 2015;125:3819-3830. [PubMed: 26368306]

[12]. Libby P, Ridker PM and Maseri A, Inflammation and Atherosclerosis, Circulation, 2002;105:1135-1143. [PubMed: 11877368]

[13]. Pober JS and Sessa WC, Evolving functions of endothelial cells in inflammation, Nat Rev Immunol, 2007;7:803-815. [PubMed: 17893694]

[14]. Hansson GK, Inflammation, Atherosclerosis, and Coronary Artery Disease, New England Journal of Medicine, 2005;352:1685-1695. [PubMed: 15843671]

[15]. Tall AR and Yvan-Charvet L, Cholesterol, inflammation and innate immunity, Nat Rev Immunol, 2015;15:104-116. [PubMed: 25614320]

[16]. Ridker PM, Everett BM, Thuren T, et al., Antiinflammatory Therapy with Canakinumab for Atherosclerotic Disease, N Engl J Med, 2017;377:1119-1131. [PubMed: 28845751]

[17]. Morita M, Ohneda O, Yamashita T, et al., HLF/HIF-2alpha is a key factor in retinopathy of prematurity in association with erythropoietin, The EMBO journal, 2003;22:1134-1146. [PubMed: 12606578]

[18]. Lu H, Fan Y, Qiao C, et al., TFEB inhibits endothelial cell inflammation and reduces atherosclerosis, Science Signaling, 2017;10.

[19]. Jawien J, Nastalek P and Korbut R, Mouse models of experimental atherosclerosis, Journal of physiology and pharmacology, 2004;55:503-517. [PubMed: 15381823] 
[20]. Gräfe M, Auch-Schwelk W, Zakrzewicz A, et al., Angiotensin II-induced leukocyte adhesion on human coronary endothelial cells is mediated by E-selectin, Circulation Research, 1997;81:804811. [PubMed: 9351454]

[21]. Ohta H, Wada H, Niwa T, et al., Disruption of tumor necrosis factor-a gene diminishes the development of atherosclerosis in ApoE-deficient mice, Atherosclerosis, 2005;180:11-17. [PubMed: 15823270]

[22]. Moyer CF, Sajuthi D, Tulli H, et al., Synthesis of IL-1 alpha and IL-1 beta by arterial cells in atherosclerosis, The American Journal of Pathology, 1991;138:951-960. [PubMed: 2012178]

[23]. Sobczak M, Dargatz J and Chrzanowska-Wodnicka M, Isolation and culture of pulmonary endothelial cells from neonatal mice, JoVE (Journal of Visualized Experiments), 2010:e2316e2316.

[24]. Wang J-M, Chen AF and Zhang K, Isolation and Primary Culture of Mouse Aortic Endothelial Cells, JoVE (Journal of Visualized Experiments), 2016:e52965-e52965.

[25]. Schindler U and Baichwal VR, Three NF-kappa B binding sites in the human E-selectin gene required for maximal tumor necrosis factor alpha-induced expression, Molecular and cellular biology, 1994;14:5820-5831. [PubMed: 7520526]

[26]. O'Neill LA and Kaltschmidt C, NF-kB: a crucial transcription factor for glial and neuronal cell function, Trends in neurosciences, 1997;20:252-258. [PubMed: 9185306]

[27]. Zhou Z, Connell MC and MacEwan DJ, TNFR1-induced NF- $\kappa$ B, but not ERK, p38MAPK or JNK activation, mediates TNF-induced ICAM-1 and VCAM-1 expression on endothelial cells, Cellular signalling, 2007;19:1238-1248. [PubMed: 17292586]

[28]. Garcia JGN, Liu F, Verin AD, et al., Sphingosine 1-phosphate promotes endothelial cell barrier integrity by Edg-dependent cytoskeletal rearrangement, Journal of Clinical Investigation, 2001;108:689-701. [PubMed: 11544274]

[29]. Iwaya C, Kitajima H, Yamamoto K, et al., DNA methylation of the Klf14 gene region in whole blood cells provides prediction for the chronic inflammation in the adipose tissue, Biochem Biophys Res Commun, 2018;497:908-915. [PubMed: 29425818]

[30]. Israël A, The IKK Complex, a Central Regulator of NF- $\kappa B$ Activation, Cold Spring Harbor Perspectives in Biology, 2010;2:a000158. [PubMed: 20300203]

[31]. Napetschnig J and Wu H, Molecular Basis of NF- $\mathrm{KB}$ Signaling, Annual Review of Biophysics, 2013;42:443-468.

[32]. Ashrafian H, Horowitz JD and Frenneaux MP, Perhexiline, Cardiovascular Therapeutics, 2007;25:76-97.

[33]. Gu L, Findley HW and Zhou M, MDM2 induces NF- $\kappa B / p 65$ expression transcriptionally through Sp1-binding sites: a novel, p53-independent role of MDM2 in doxorubicin resistance in acute lymphoblastic leukemia, Blood, 2002;99:3367-3375. [PubMed: 11964305]

[34]. Kim M-Y, Koh D-I, Choi W-I, et al., ZBTB2 increases PDK4 expression by transcriptional repression of RelA/p65, Nucleic Acids Research, 2015;43:1609-1625. [PubMed: 25609694]

[35]. SenBanerjee S, Lin Z, Atkins GB, et al., KLF2 Is a novel transcriptional regulator of endothelial proinflammatory activation, The Journal of experimental medicine, 2004;199:1305-1315. [PubMed: 15136591]

[36]. Hamik A, Lin Z, Kumar A, et al., Kruppel-like Factor 4 Regulates Endothelial Inflammation, Journal of Biological Chemistry, 2007;282:13769-13779. [PubMed: 17339326]

[37]. Fan Y, Guo Y, Zhang J, et al., Kruppel-like factor-11, a transcription factor involved in diabetes mellitus, suppresses endothelial cell activation via the nuclear factor-kappaB signaling pathway, Arterioscler Thromb Vasc Biol, 2012;32:2981-2988. [PubMed: 23042817]

[38]. Hao JS, Zhu CJ, Yan BY, et al., Stimulation of KLF14/PLK1 pathway by thrombin signaling potentiates endothelial dysfunction in Type 2 diabetes mellitus, Biomed Pharmacother, 2018;99:859-866. [PubMed: 29710485]

[39]. Bolognesi B and Lehner B, Reaching the limit, eLife, 2018;7:e39804. [PubMed: 30095407]

[40]. Reiner Z, Statins in the primary prevention of cardiovascular disease, Nat Rev Cardiol, 2013;10:453-464. [PubMed: 23736519] 
[41]. Giugliano RP and Sabatine MS, Are PCSK9 Inhibitors the Next Breakthrough in the Cardiovascular Field?, Journal of the American College of Cardiology, 2015;65:2638-2651. [PubMed: 26088304]

[42]. Ahn $\mathrm{CH}$ and Choi SH, New drugs for treating dyslipidemia: beyond statins, Diabetes \& metabolism journal, 2015;39:87-94. [PubMed: 25922802] 
A

DAPI CD31 KLF14 Merge

B

C

PBS

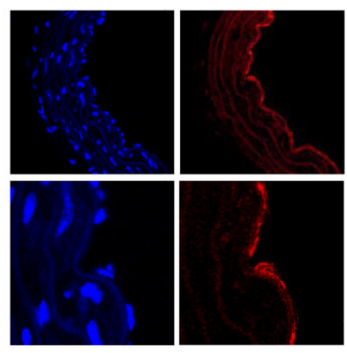

LPS

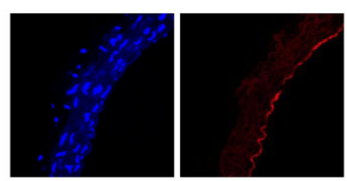

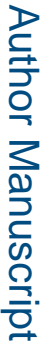

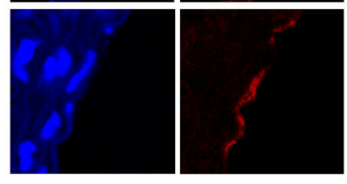

DAPI

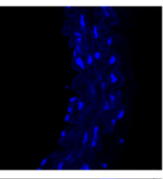

CD
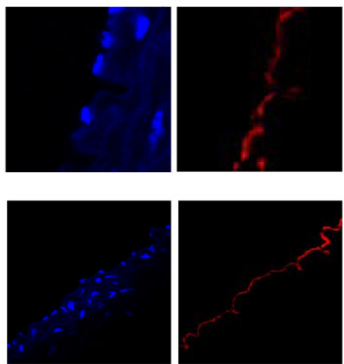

WD

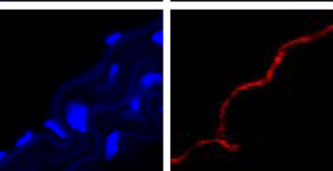

CD31
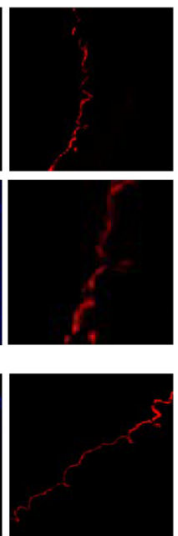
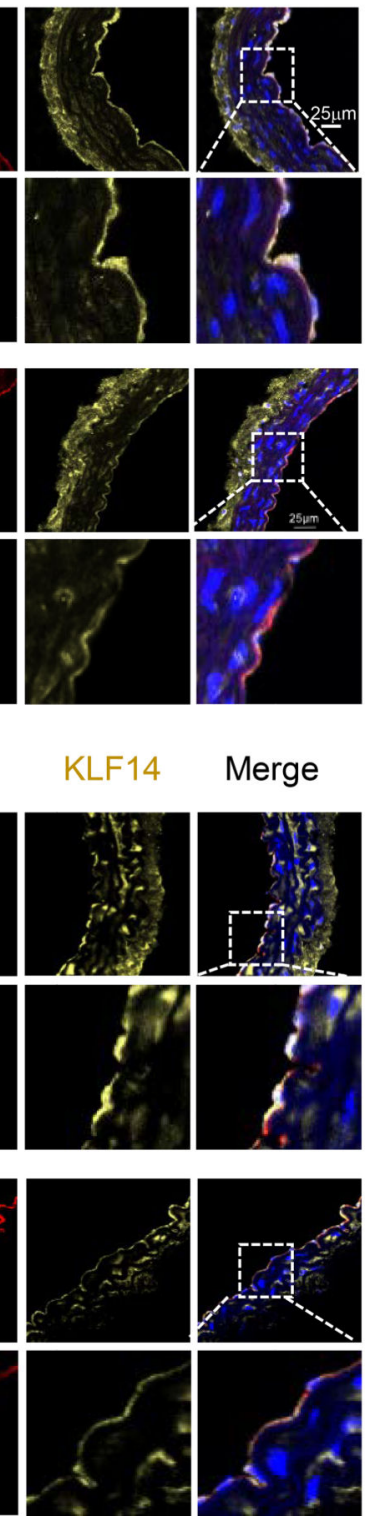

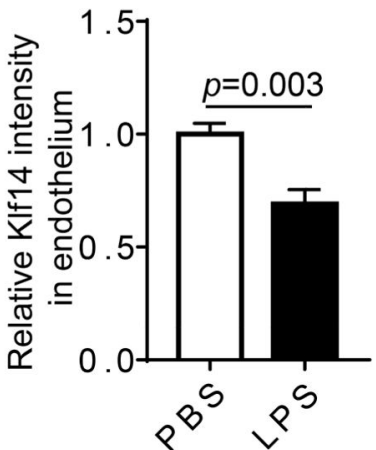

E
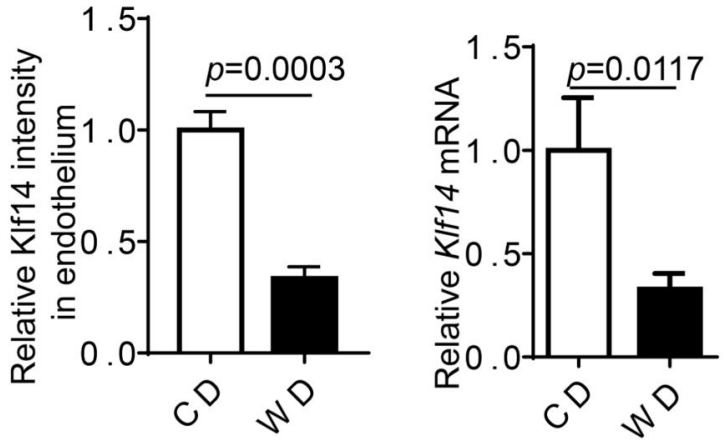

H
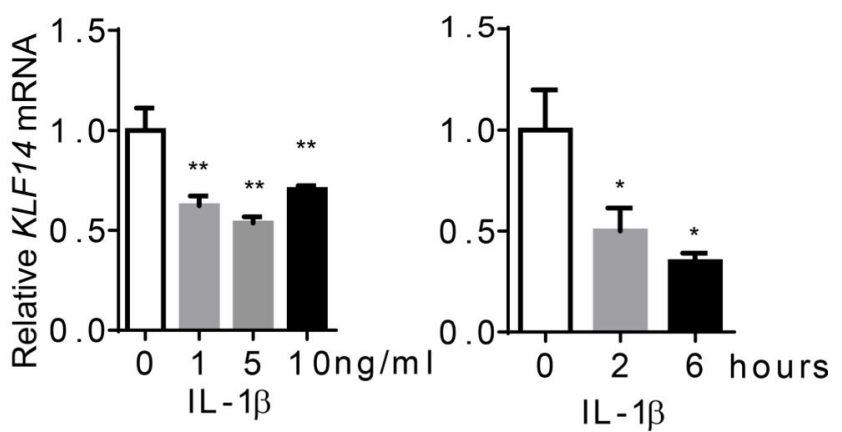

Fig. 1. Endothelial KLF14 is down-regulated in both acute and chronic inflammatory conditions. (A-C) Eight-week-old male C57BL/6J mice were treated with PBS or lipopolysaccharide (LPS, $20 \mathrm{mg} / \mathrm{kg}$ ) via intraperitoneal injection. Six hours later, aortas were collected. (D-F) Six-week-old C57BL/6J mice were fed chow diet (CD) or western diet (WD) for three months. Aortas were collected. Adventitia was carefully removed from aortas in both conditions before tissue mRNA was extracted. Expression of KLF14 was determined by immunofluorescence and confocal images (A and D) and qRT-PCR (C and F). (G) Human umbilical vein endothelial cells (HUVECs) were stimulated with the indicated concentration of interleukin $1 \beta$ (IL-1 $\beta$ ) for 6 hours and Klf14 levels were detected by qRT-PCR. (H) IL-1 $\beta$ $(10 \mathrm{ng} / \mathrm{ml})$ was used to stimulate HUVECs in a time course experiment and KLF14 levels 
were detected by qRT-PCR. $\left({ }^{*} p<0.05 ;{ }^{* *} p<0.01\right.$, compared to PBS, CD, or no treatment group.) 


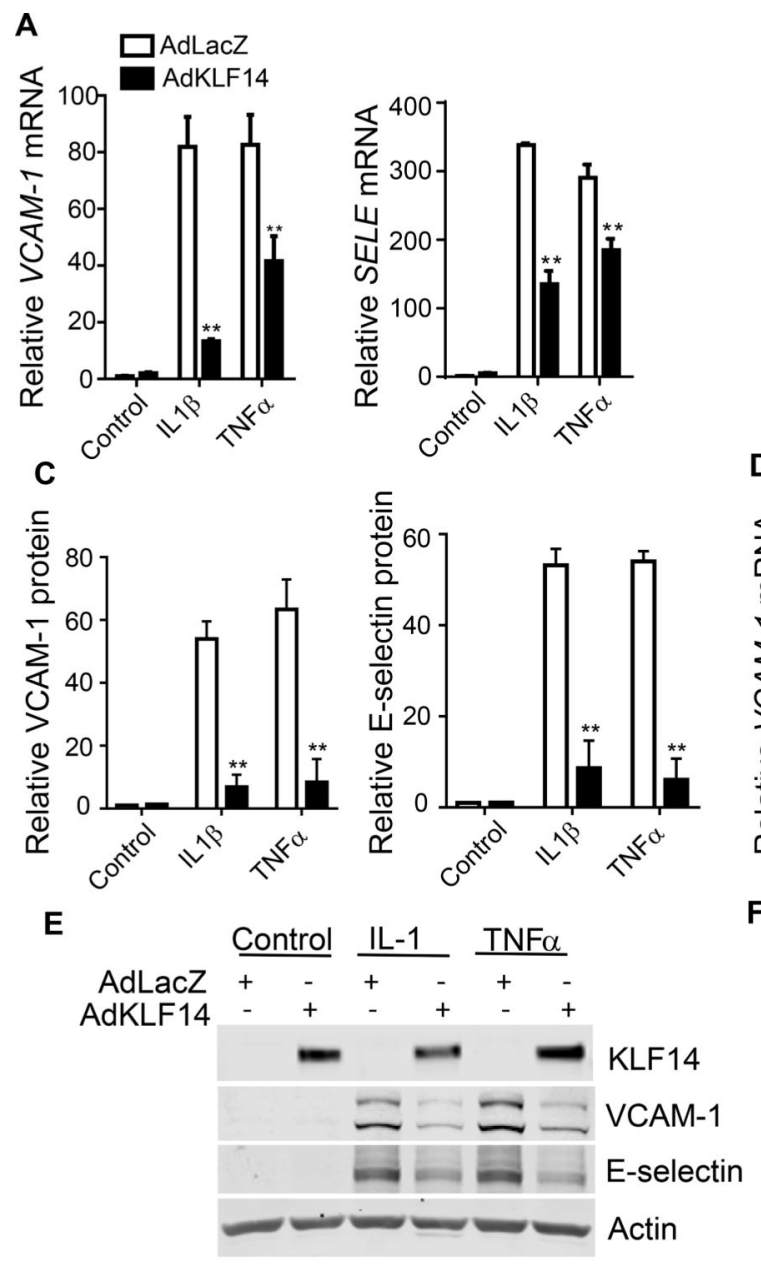

G

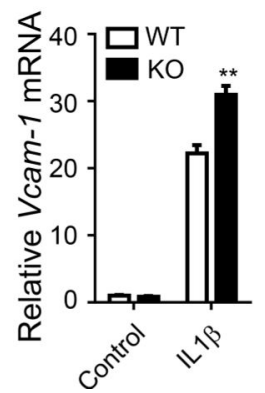

H

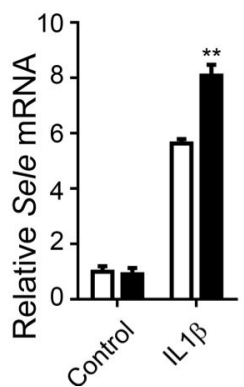

B

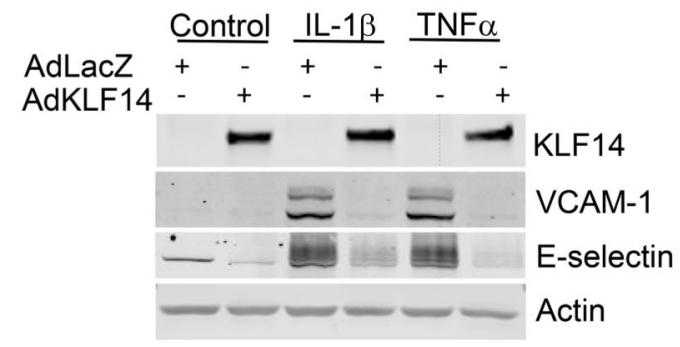

D

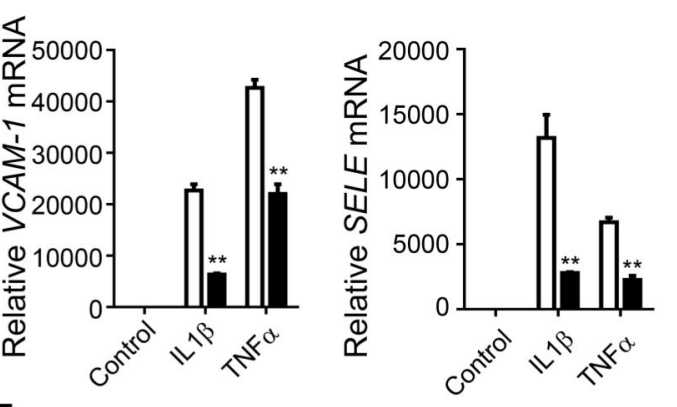

F

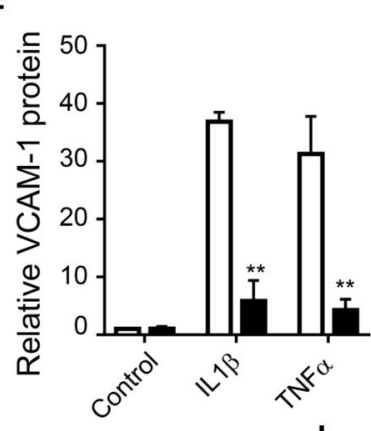

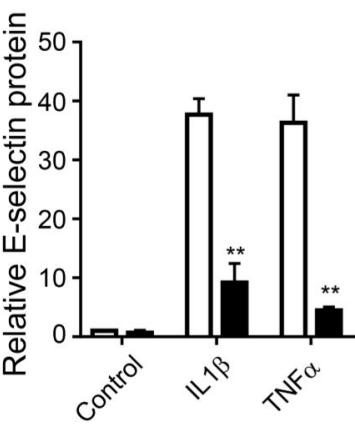

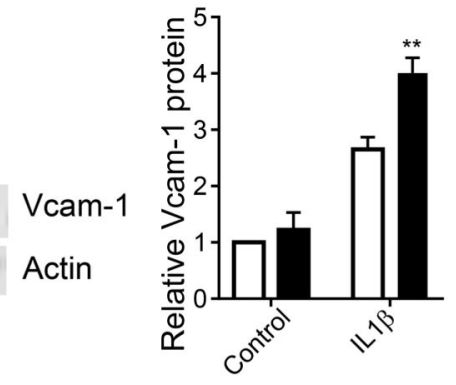

Fig. 2. KLF14 inhibits pro-inflammatory cytokine-induced expression of inflammatory markers in endothelial cells.

HCAECs (A-C) and HUVECs (D-F) were infected with 10 MOI of adenovirus encoding lacZ (AdLaZ) or human KLF14 (AdKLF14) for 48 hours and then stimulated with $5 \mathrm{ng} / \mathrm{ml}$ IL- $1 \beta$ or $2 \mathrm{ng} / \mathrm{ml}$ TNFa respectively for 4 hours. Endothelial inflammatory markers $V C A M-1$ and $S E L E$ (encoding E-selectin) mRNA abundance was determined by qRT-PCR (A and D). VCAM-1 and E-selectin protein levels were determined by western blot (B and E) and quantitatively analyzed (C and F). (G-I) Mouse pulmonary endothelial cells were isolated from Klf14 global KO mice (KO) and their wild type littermates (WT) and 
stimulated with $20 \mathrm{ng} / \mathrm{ml} \mathrm{IL-1 \beta}$ for 4 hours. (G) Vcam- 1 and Sele mRNA abundance was determined by qRT-PCR. Vcam-1 protein levels $(\mathrm{H})$ were determined by western blot and quantitatively analyzed (I). ${ }^{*} p<0.01$, compared to AdLacZ or WT group. 
A

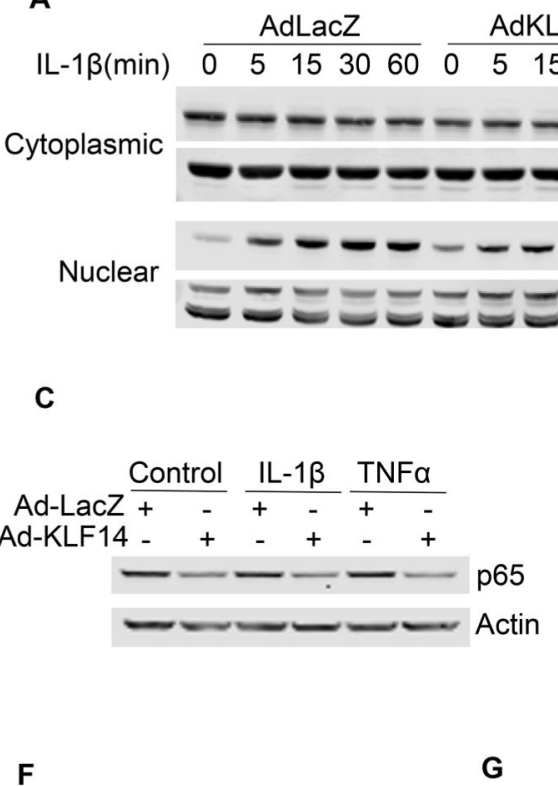

$\mathbf{F}$
B

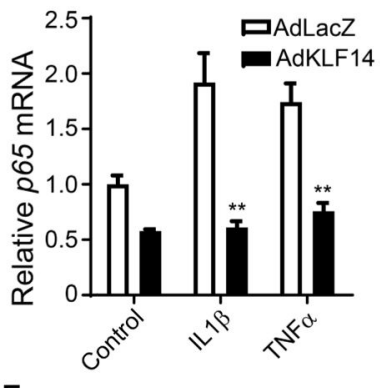

$\mathbf{E}$

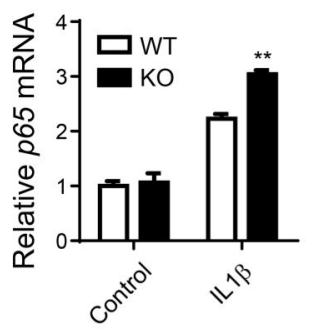

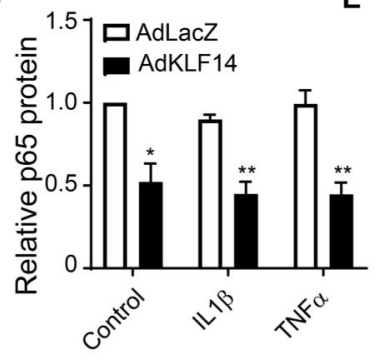

H

H

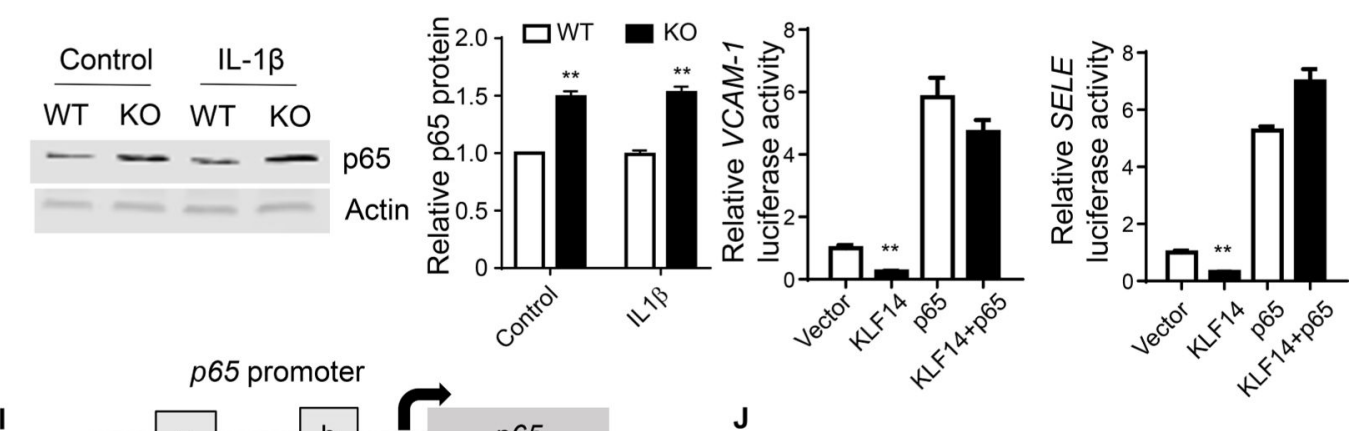

I

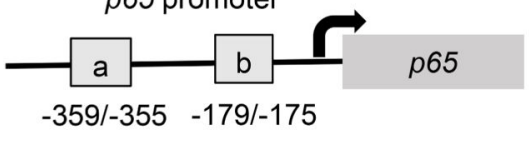

J

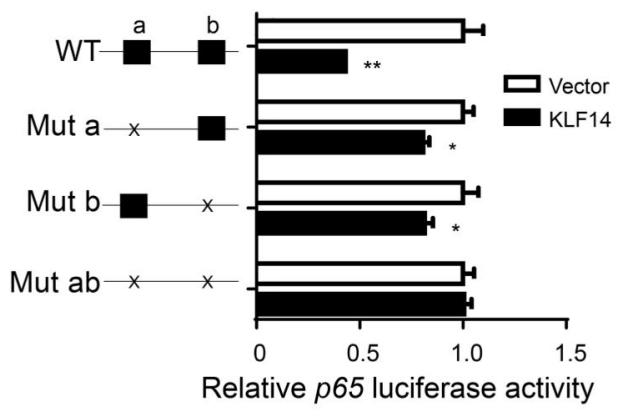

Fig. 3. KLF14 inhibits p65 transcription.

(A) HUVECs were infected with 10 MOI of AdLacZ or AdKLF14 for 48 hours and then stimulated with $5 \mathrm{ng} / \mathrm{ml} \mathrm{IL-1 \beta}$ for the indicated time. Cytoplasmic and nuclear p65 protein levels were determined by western blot. (B-D) HUVECs were infected with $10 \mathrm{MOI}$ of AdLacZ or AdKLF14 for 48 hours and then stimulated with $5 \mathrm{ng} / \mathrm{ml} \mathrm{IL-1 \beta}$ or $2 \mathrm{ng} / \mathrm{ml} \mathrm{TNFa}$ for 4 hours. p65 mRNA abundance was determined by qRT-PCR (B). Total p65 protein levels were determined by western blot $(\mathrm{C}$ and $\mathrm{D})$. Primary culture of mouse pulmonary endothelial cells from WT or Klf14 deficient mice were treated with IL-1 $\beta$ ( $20 \mathrm{ng} / \mathrm{ml})$ for 4 
hours. p65 expression was determined by qRT-PCR (E) and western blot (F and G). Ad293 cells were co-transfected with $V C A M-1$ or $S E L E$ promoter driving luciferase reporter plasmid with pcDNA3.1 (vector), KLF14 or p65 plasmid. After 24 hours, the transfected cells were stimulated with $10 \mathrm{ng} / \mathrm{ml} \mathrm{TNFa}$ for 16 hours and then luciferase activities were detected (H). Renilla-null luciferase activity served as the internal control. HUVECs were infected with 10 MOI of AdLacZ or flag tagged KLF14 for 48 hours. The binding of KLF14 protein to box a and box b was determined by ChIP assay (I). Ad293 cells were cotransfected with WT or mutated p65 promoter luciferase reporter plasmid and vector or KLF14 plasmid for 24 hours and then stimulated with $10 \mathrm{ng} / \mathrm{ml} \mathrm{TNFa}$ for 16 hours and then luciferase activities were detected (J). Renilla-null luciferase activity served as the internal control. Left panel shows the mutant pattern of the CACCC binding boxes on the p65 promoter. The right panel shows the corresponding $\mathrm{p} 65$ promoter luciferase activity in response to KLF14. ${ }^{*} p<0.05$; $* * p 0.01$, compared to AdLacZ, WT or vector group. 
A
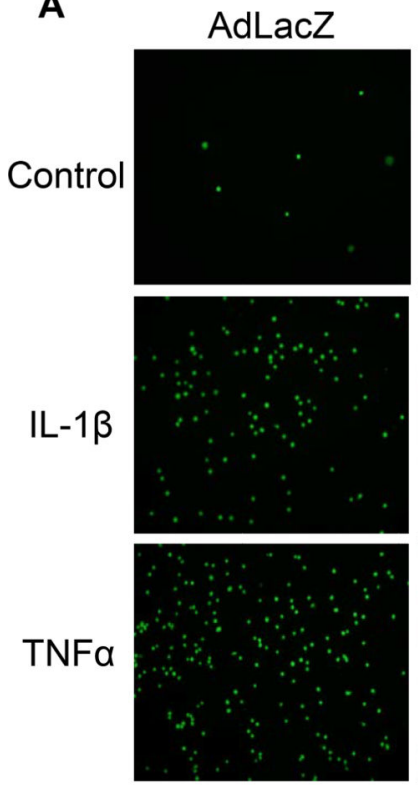

C

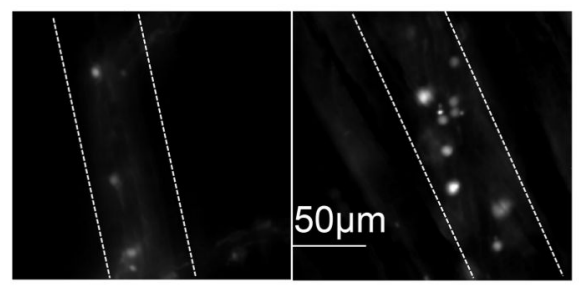

WT

\section{AdKLF14}
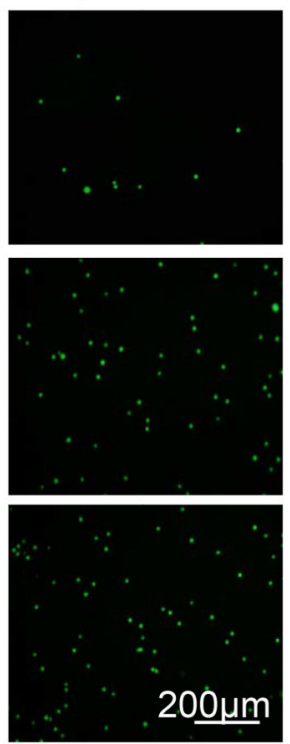

$\mathrm{EC} \mathrm{KO}$

\section{B}

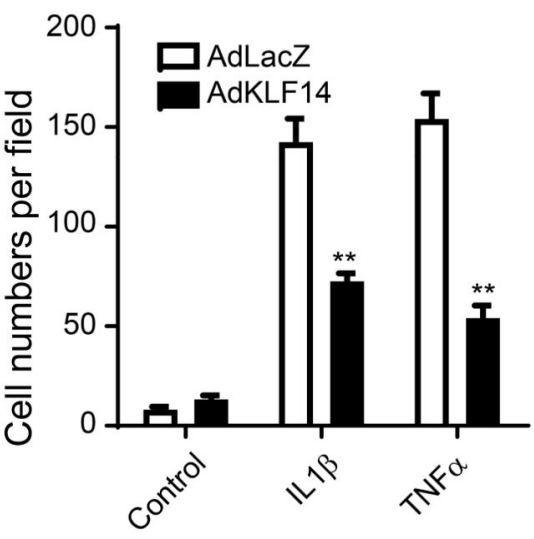

D

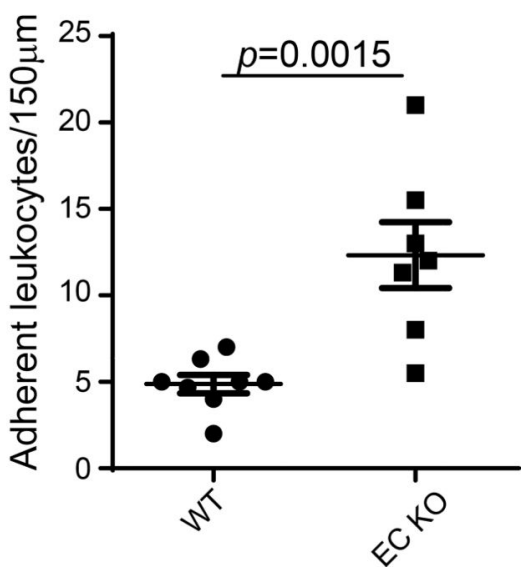

Fig. 4. KLF14 inhibits leukocyte-endothelial adhesion.

(A) and (B) HUVECs were infected with 10 MOI AdLacZ or AdKLF14 for 48 hours, stimulated with $5 \mathrm{ng} / \mathrm{ml} \mathrm{IL}-1 \beta$ or $2 \mathrm{ng} / \mathrm{ml} \mathrm{TNFa}$ for 6 hours and then co-incubated with fluorescence-labelled THP-1 cells. Adherent THP-1 cells were observed (A) and counted (B) under a fluorescence microscope. (A) Green spots represent adherent THP-1 cells. Eight weeks old male KLF14 EC KO mice and littermate wild type mice were administered intraperitoneal injection of $5 \mathrm{mg} / \mathrm{kg}$ LPS. Four hours later, leukocyte-endothelial adhesion was observed under a intravital microscope (C). Dashed lines represent vessel walls. White spots represented adherent leukocytes. Scale bar, $50 \mu \mathrm{m}$. (D) The adhesion of leukocytes on vessel walls was quantified. $* * p<0.01$, compared to AdLacZ or WT group. 

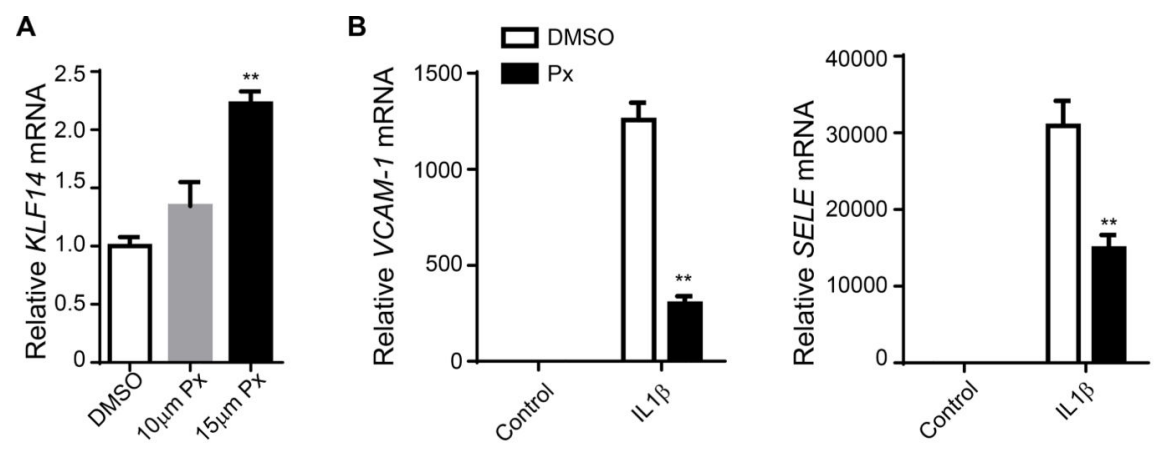

C

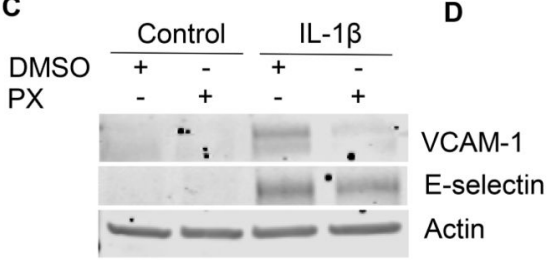

E

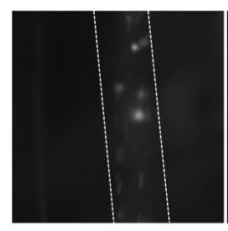

WT

DMSO

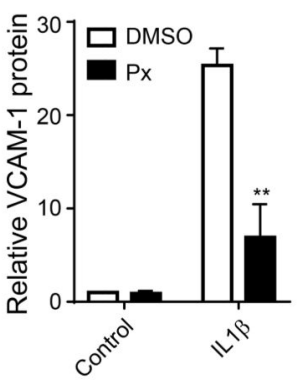

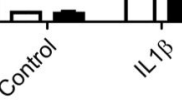

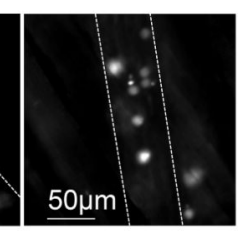

EC KO

$\mathrm{Px}$

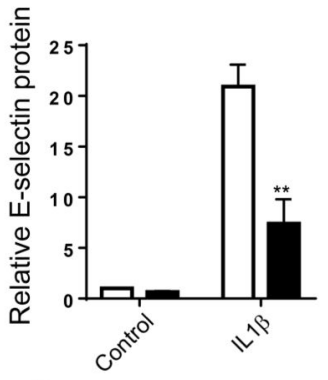

F

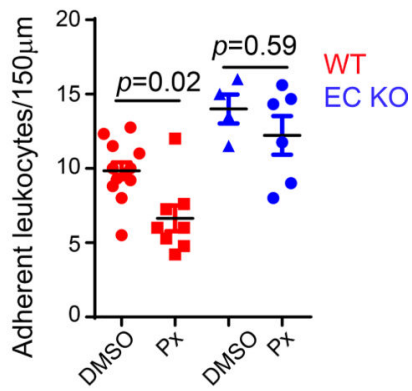

G

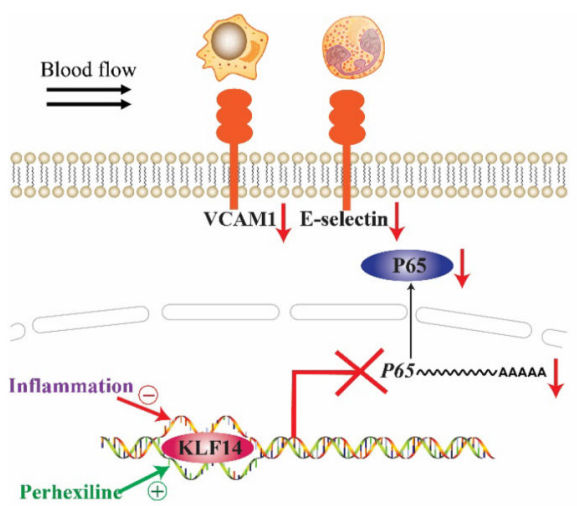

Fig. 5. Perhexiline inhibits endothelial inflammation in an endothelial KLF14 dependent manner. HUVECs were treated with DMSO or the indicated concentration of perhexiline maleate salt (Px) for 20 hours. KLF14 expression was determined by qRT-PCR (A). (B-D) HUVECs were pretreated with DMSO or $15 \mu \mathrm{M}$ perhexiline maleate salt for 20 hours and then stimulated with $5 \mathrm{ng} / \mathrm{ml} \mathrm{IL}-1 \beta$ for 4 hours. VCAM-1 and SELE mRNA abundance was determined by qRT-PCR (B). VCAM-1 and E-selectin protein levels were determined by western blot (C) and (D). (E and F) Eight-week-old male wild type mice and KIf14 EC KO mice were injected intraperitoneally with DMSO or perhexiline maleate salt $(10 \mathrm{mg} / \mathrm{kg} / \mathrm{d})$ 
every other day for 5 days before they were administered (intraperitoneally) $10 \mathrm{mg} / \mathrm{kg}$ LPS for 4 hours. Leukocyte-endothelial adhesion was observed under an intravital microscope (E) and quantified (F). (G) Schematic diagram showing the effect and mechanism of KLF14 in endothelial cell inflammation. (** $p<0.01$, compared to DMSO or WT group.) 